\title{
Stabilization of Highly Concentrated Colloidal Suspension of Pristine Metallic Nanoparticles
}

\author{
Nirmal Kumar Katiyar' ${ }^{1}(0000-0001-8795-7857)$, Krishanu Biswas $^{1^{*}}$, Chandra Sekhar
}

Tiwary $^{2 *(0000-0001-9760-9768), ~ L e o n a r d o ~ D a n t a s ~ M a c h a d o ~}{ }^{\mathbf{3}}$, Raju Kumar Gupta ${ }^{\mathbf{4 , 5}}$ (0000-0002-

5537-8057)

${ }^{1}$ Department of Material Science \& Engineering, Indian Institute of Technology Kanpur -208016, India ${ }^{2}$ Department of Metallurgical and Material Engineering, Indian Institute of Technology Kharagpur, West Bengal-721302, India

${ }^{3}$ Departamento de FísicaTeórica e Experimental, Universidade Federal do Rio Grande do Norte, NatalRN, 59072-970, Brazil.

${ }^{4}$ Department of Chemical Engineering, Indian Institute of Technology Kanpur -208016, India

${ }^{5}$ Center for Nanosciences and Center for Environmental Science and Engineering, Indian Institute of Technology Kanpur -208016, India

\begin{abstract}
The colloidal suspension containing a high concentration of metallic nanoparticles (NPs) find potential applications in flexible electronic printing, nanofluids, healthcare, and antifouling coating, etc. Here, we demonstrate a generic, easily scalable, simple and contamination free cryogenic temperature grinding method, which can effectively be used to prepare pristine NPs and can be stabilized in polar liquids in high-concentrations. These surfactant free pristine nanoparticles have been found to remain dispersed in different polar liquids $\left(\mathrm{CH}_{3} \mathrm{OH}, \mathrm{C}_{2} \mathrm{H}_{5} \mathrm{OH}\right.$, Glycol, etc.) for weeks. The long term stability of the nanoparticles in these liquids has been investigated using zeta potentials, in-situ FTIR spectroscopy indicating electrostatic stabilization for ultra pure, surfactant free NPs. Furthermore, stabilization of the NPs has been probed detailed calculation using DLVO theory as well as atomistic molecular dynamic simulation (MD). Experimental measurements along with theoretical calculation categorically indicate the electrostatic energy is helping these nanoparticles to be stabilized in a polar liquid.
\end{abstract}

Keywords: Nanoparticle colloids, Metallic NPs, Microscopy, Zeta potentials, DLVO, MD

simulation.

*e mail-kbiswas@iitk.ac.in, cst.iisc@gmail.com, 


\section{Introduction}

Metallic nanoparticles (NPs) can be made to free stand in colloidal suspension due to two distinctly different mechanisms, depending on whether NPs are capped by organic molecules or native $\mathrm{e}^{1-3}$. It is well known that the former is stabilized in the colloids due to steric forces because of the presence of the capping agent. On the other hand, the uncapped NPs are electrostatically stabilized due to the native surfaces ${ }^{4-6}$. Although the capping agents help in the steric stabilization of the NPs in liquids, they reduce the direct interaction of NPs with each other as well as the space for preferential adsorptions of ions/molecules on the surfaces of the NPs. It also modifies the surface properties of the NPs drastically limiting the application of the NPs ${ }^{7-12}$. However, the uncapped NPs provide more freedom to engineer the surfaces and hence properties, and they can find more applications. These applications require large scale synthesis of the surfactant of free uncapped metallic NPs, which can be stabilized in polar liquids to form a colloidal solution containing a high concentration of NPs.

The last two decades have witnessed extensive research to develop processing techniques suitable to prepare free standing ultra-pure metallic NPs to fulfill the requirements for different applications. These research activities have categorically indicated that grinding/milling at cryogenic temperature $(<123 \mathrm{~K})$ can efficiently be utilized to prepare surfactant free metallic NPs is large quantity ${ }^{13}$, which can subsequently be utilized for preparation of colloids in various liquids $^{13-15}$. However, the concentration of the NPs in the colloids is considered to be the most important aspect for applications in the flexible electronic printing ${ }^{16}$, nanofluids ${ }^{17-18}$, optical sensing ${ }^{19}$, bio-imaging ${ }^{20}$ or even antifouling coatings ${ }^{21}$ for sea borne vehicles. Till date, only a limited amount/concentration of loading of NPs in the solvent could be achieved ${ }^{22}$. This limitation is ascribed to the usages of capped NPs to prepare a colloid suspension in which steric 
stabilization of the NPs is considered to be the dominant mechanism [7-8]. To extend the applications as indicated earlier, it is thus important to control the concentration level of the NPs in the colloidal solvent so that a high amount of loading can be achieved. However, to achieve the goal, it is required to understand the mechanism responsible for the stabilization of high concentration of surfactant free NPs in various polar liquids.

In the present investigation, different metallic $\mathrm{NPs}(\mathrm{Al}, \mathrm{Ag}, \mathrm{Cu}, \mathrm{Fe}, \mathrm{Zn})$ have been synthesized using cryomilling. Subsequently, NPs have been added to various polar solvents $\left(\mathrm{CH}_{3} \mathrm{OH}\right.$, $\mathrm{C}_{2} \mathrm{H}_{5} \mathrm{OH}$, Glycol, and Benzene) in different concentration levels to probe the stability of the NPs in the liquids. These surfactant free NPs can be stabilized in polar liquids for weeks or even months for some metals (Al, Zn). Therefore, the stabilization of NPs in the solvents have been probed by studying the interaction of the native surface of NPs with polar solvent using DLVO (Derjaguin, Landau, Verwey, and Overbeek) theory ${ }^{23}$. Furthermore, detailed MD simulation has been performed to understand atomic scale mechanism for stabilization of metallic NPs in the liquids. These calculations have been backed up by in-situ FTIR and Zeta potentials measurements, probing the interaction of the native surface with the molecules of the liquids.

\section{Experimental details}

The metallic nanoparticles ( $\mathrm{Al}, \mathrm{Ag}, \mathrm{Cu}, \mathrm{Zn}$, and $\mathrm{Fe}$ ) have been prepared using a custom built cryomill. The milling has been carried out using the cryomill designed in such a fashion that the coolant (Liquid $\mathrm{N}_{2}$ ) and the milling powder could never be mixed. This has been done to protect the metallic NPs from nitridation. The mill is a single ball vibratory mill with attachments for temperature control, ball motion tracking as well as amplitude control. The vibration amplitude of $1.5 \mathrm{~mm}$ has been utilized throughout milling of the nanoparticles. The detailed design of 
cryomill has been reported elsewhere ${ }^{24}$. The powder and ball were charged in the vial surrounded by liquid nitrogen. The powders were milled for seven hours and temperature was maintained at $-160 \pm 10{ }^{\circ} \mathrm{C}$ throughout milling by the intermittent pouring of $\mathrm{LN}_{2}$ in the annular chamber. During milling, argon gas was purged inside the milling chamber at a constant rate of $2 \mathrm{~L} / \mathrm{h}$ to ensure that the as-prepared NPs were not oxidized. Also, extra care was taken to protect the milled powder from oxidation. The extremely low temperature also protects the milled powder from oxidation and nitridation. Afterward, the milled powder was immediately poured in the ultrapure methanol and sonicated for 10 minutes to ensure the dispersion of NPs. The concentration of nanoparticles in the liquids has been back calculated to avoid any exposure of NPs surfaces with environmental gases. The interactions of NPs with the solvent were investigated using FTIR (Fourier Transform Infra-Red) spectroscopy as well as Raman spectroscopy in the liquid phase with varying sonication time (0 to 30 minutes). Further, Zeta potential of the dispersed nanoparticles at room temperature has been measured by using Malvern zeta-sizer Nano ZS90 with varying $\mathrm{pH}$. The $\mathrm{pH}$ was measured using Mettler Toledo $\mathrm{pH}$ meter, and the the similar concept has been applied as for aqueous solution. It was also adjusted using $\mathrm{NH}_{4} \mathrm{OH}$ and $\mathrm{CH}_{3} \mathrm{COOH}$ as weak base and acid respectively. The microstructure and size distribution of the nanoparticles has been obtained by analyzing HRTEM (high resolution transmission electron microscope, FEI, Titan $\mathrm{G}^{2} 60$ operated at $300 \mathrm{kV}$ ) images. The UV-visible spectrophotometer (Thermo scientific evolution 201) has been used to study surface plasmon resonance characteristics of the metal nanoparticles.

\section{Simulation details}

We carried out fully atomistic molecular dynamics (MD) simulations. In the simulations, the Reactive force field (ReaxFF) ${ }^{25-26}$ was employed, which was implemented in the LAMMPS MD 
package ${ }^{27}$. Two key advantages of ReaxFF are its capability to describe bond formation/dissociation and determine atomic charges from the chemical environment (charge equilibration $)^{28}$. Due to the latter feature, changes in the atomic neighborhood are accompanied by changes in atomic partial charge values. The $\mathrm{Cu}$ NPs were immersed in methanol, and then a long equilibration process was performed at room temperature, as consistent with experiments with methanol as solvent. The system investigated in the reactive molecular dynamics simulations consisted of two copper nanoparticles (each with a $2 \mathrm{~nm}$ radius and containing 456 atoms) and many solvent molecules. To obtain a $1 \mathrm{~nm}$ horizontal separation between particles, 946 methanol molecules were used.

Similarly, for a $3.5 \mathrm{~nm}$ separation, 1966 methanol molecules were utilized. Initially, the solvent molecules were far apart and then a long equilibration process was carried out to obtain a solution with copper nanoparticles immersed in the methanol that was in equilibrium at $300 \mathrm{~K}$. The six steps in the process used are described below:

1. 1000 steps of conjugate gradient minimization were carried out;

2. The initial velocities for methanol atoms were attributed to $300 \mathrm{~K}$, that were consistent with room temperature;

3. The atoms on the surface of the NPs were kept fixed, and calculations were performed 500000 steps of equilibration allowing only the movement of solvent atoms. This process was carried out in the NPT ensemble using a Nosé-Hoover thermostat and barostat to set the temperature to $300 \mathrm{~K}$ and the pressure to $1000 \mathrm{~atm}$. Initial pressure was set high to bring the dispersed methanol atoms close enough to interact;

4. The initial velocities were set for copper atoms that were consistent with the temperature of $(300 \mathrm{~K})$ 
5. Then another 500000 steps of equilibration were performed in the NPT. However, all atoms were set free to evolve. The temperature was fixed at $300 \mathrm{~K}$, but the pressure was continually lowered from 1000 atm to 1 atm, once again using a Nosé-Hoover thermostat and barostat;

6. Finally, the thermostat was turned off and set the entire system free to evolve in the NVE ensemble for 1000000 steps. The data displayed in Figure 4(c) was obtained during this simulation step. The data presented in Figure 4(b) was obtained at the end of this step.

Throughout the simulations, a time step of $0.1 \mathrm{fs}\left(1 \mathrm{fs}=10^{-15} \mathrm{~s}\right)$ and periodic boundary conditions in all directions were used. We discuss larger unit cells, composed of 4 and 8 copper nanoparticles, in the Supporting Information.

\section{Results and discussion}

To probe the ability of the as synthesized NPs to form colloids in various liquids, the NPs have been dispersed in ultrapure methanol followed by ultra sonication, just after cryomilling and without any environmental exposure. It is well known that the NPs are reported to exhibit surface plasmon resonance (SPR) band, which is primarily due to collective oscillation of the valence electrons on nanoparticles surface ${ }^{29}$. The dielectric of the surrounding medium is reported to effect on peak position and intensity ${ }^{30}$. Although the size and shape also alter the peak position, it is certain that as size decreases the blue shift in the peak position is observed (red shift: peak shift to higher wavelength; blue shift: peak shift to lower wavelength). In case of cryomilling, the nanoparticles are expected to have uneven size with a roughened surface. The SPR peaks of different metals nanoparticles dispersed in methanol have been shown in Figure 1(a) with the inset showing the optical images of different NPs in methanol. The size and SPR (surface 
plasmon reasonance) peaks of the other metallic nanoparticles are summarized in Table $\mathbf{1}$. Further, the dispersion of $\mathrm{Cu}$ NPs in the methanol with varying concentration is also shown in Figure 1(b), and their corresponding UV-spectra is available in Figure 1(c), revealing the increasing intensity of absorbance due to the higher concentration of NPs. Some of metallic NPs were finely dispersed in the methanol for a longer time. Al and Zn NPs were remained dispersed in the liquid more than a month, where as $\mathrm{Cu}$ for more than 10 days, $\mathrm{Ag}$ and Fe NPs for a couple of days. It There is no evidence of any reaction between methanol and nanoparticles interface characterized by liquid phase FTIR spectra as shown in Figure 1(d). After the dispersing the metal NPs in methanol, FTIR spectra have been observed with varying ultra sonication time $(0$, 2, 5, 10, 20, 30 minutes). However, there no extra peak has been arised except the increase of the transmittance intensity, which might be due to more retention time of methanol when mixed with NPs.

Similarly, the Raman spectra have been recorded to find the possibility of any reaction over nanoparticles surface (for Al NPs has shown due to longer stability) (see Supporting Information Figure S5). However, no evidence of any reaction has been observed. The XPS measurements do not reveal evidence of oxidation and nitridation of the milled powder as shown in Figure 1(e) and Figure 1(f). The peaks at $530.93 \mathrm{eV}$ and 532.61 in Figure 1(e) are attributed to the adsorbed oxygen and $\mathrm{H}_{2} \mathrm{O}$ respectively. Therefore; no foreign contamination has been found from cryomilling during preparation.

The morphology, size and distribution of the as prepared NPs were investigated using HRTEM. Figure 2, shows TEM bright field image of Al NPs (average size $=6 \pm 3 \mathrm{~nm}$ ). The inset reveals the high-resolution images. HRTEM images of Ag NPs (average size $=6 \pm 2 \mathrm{~nm}$ ); Cu NPs $($ average size $=7 \pm 2 \mathrm{~nm}) ;$ Fe NPs (average size $=8 \pm 3 \mathrm{~nm})$ and Zn NPs (average size $3 \pm 1 \mathrm{~nm}$ ) are 
shown (type of NPs mentioned on the image in Figure 2). It is to be noted that, the size of the NPs was estimated using at least 500 of NPs from bright field images so that a reasonable statistic could be achieved (see Supporting information for details, Figure S6).

The present investigation categorically shows that the metallic NPs, prepared using cryomilling are uniformly dispersed in polar liquids for long duration. The stabilization of metallic nanoparticles in these liquids needs explanation. The stabilization of the native NPs (without any capping agent) can be due to two important aspects; electrostatic stabilization, and possible electrochemical interaction of the atoms on the surface of the nanoparticles with molecules of the solvents. The NPs prepared via top-down approach at cryo temperature, would have native/virgin surface consisting of unsatisfied dangling bonds. Additionally, the effect of gravitational forces will be the negligible as the metallic NPs are in the ultra-refined $(<10 \mathrm{~nm})$ range. The electrostatic interactions of the nanoparticles in the solutions are two types; NP-solvent interactions and NP-NP interactions. It is well known that the alcohol molecules can be over the clean metallic surface. The dangling bonds existing over the surface are free, can easily allow the methanol molecules physisorbed to reach electrostatic equilibrium. As a result, the electrical double layer as shown in Figure 3 (a) can be formed ${ }^{31-33}$. The nature of the electrical double layer (EDL) on the surface of metallic nanoparticles dictates the interactions among particles. However, the stability of the nanoparticles in a solution is directly related to the potential barrier, as shown schematically in Figure 3(b). The nanoparticles can freely move in the solvent due to Brownian motion and repel each other due to similar charge at slipping plane (layer moves along with nanoparticles). The repulsion force between two nanoparticles decides the dispersion stability, which is induced by NPs-NPs and NPs-Solvent interaction and create an energy barrier to overcome for agglomeration. The resultant theoretical barrier (addition of Van der Waals 
attraction and electrostatic repulsion) has been calculated using DLVO theory (see supporting information for detailed calculation).

Considering two metallic nanoparticles dispersed in methanol solvent, the calculated force profile is shown in Figure 3(c), clearly illustrating the dominance of repulsive force over Van der Waals force of attraction as inter-particle separation reduces and a peak is observed approximately at $2 \mathrm{~nm}$ distance for various nanoparticles. As shown in Figure 3(c), the height of the barrier varies for different NPs, which can directly be related to the stability of the dispersion. Higher the value of the potential barrier, it is less likely to be overcome by the kinetic energy produced by Brownian motion and nanoparticles remain in a free-standing state and well dispersed in the liquid for a longer time. According to our calculations, Cu NPs and Ag NPs have the highest and lowest potential barrier respectively, which is directly related to their stability in methanol, as observed in the experiments.

The potential or charge at the slipping plane is called zeta potentials $(\zeta)$. It has been reported that the zeta potential $> \pm 30 \mathrm{mV}$ can be considered as a threshold value for dispersion stability. Therefore, the stability of the dispersion in the present investigation has been investigated using zeta potential measurement with varying $\mathrm{pH}$ as shown in Figure 3(d). The $\mathrm{Cu}$ and $\mathrm{Zn} \mathrm{NPs}$ at neutral $\mathrm{pH}$ revealed a zeta potential $>30 \mathrm{mV}$, indicating $\mathrm{Cu}$ and $\mathrm{Zn}$ are highly stable. Similarly, shown in Figure 3(d) Cu NPs remain dispersed for 10 days and Zn NPs (longer than a month) as well $\mathrm{Al}(+23 \mathrm{mV}$ at $\mathrm{pH} 7)$ remain to disperse for a month. However, $\mathrm{Ag}(-20 \mathrm{mV}$ at $7 \mathrm{pH})$ and $\mathrm{Fe}$ NPs have dispersion strength for approximate a day or two. This can further be explained by NPNP interaction in the solvent (methanol) using atomistic molecular dynamics (MD) simulations 
and DLVO theory (Movie showing $\mathrm{Cu}$ NPs interacting with methanol molecules is included in the supporting information).

To investigate the distribution of charges near the native surface of a metallic nanoparticle (copper as a representative) immersed in methanol, a detailed MD simulation has been carried and the results are shown in Figure 4. A snapshot of the atomic configuration after equilibration is presented in Figure 4(a), for an NP separation distance of $1 \mathrm{~nm}$. Notice most solvent atoms are transparent, with the exception of those located within $2.5-3.0 \AA$ of the nanoparticle surface. To determine the distance of a given solvent atom to the NP surface, its distance to the nearest $\mathrm{Cu}$ atom was calculated. Qualitatively, near the NP, mostly oxygen atoms were found followed by hydrogen atoms, and next to a mix of carbon and hydrogen atoms. Though oxygen atoms adhered strongly to the surface, dissociation of methanol was never observed. To quantify the observations, the average charge values were measured at varying distances from the NP surface. We divided the space around the NPs in regions $0.5 \AA$ wide, starting with one located between $1.0-1.5 \AA$ of the nanoparticle surface, and then determined the average charge value for atoms within this layer. The results for two NP separation distances are presented in Figure 4(b). The average charge at the first data point in this graph is exactly zero because there is no atom this close to the nanoparticles. Next, one can also observe high negative average values at the second layer, due to the predominance of oxygen atoms at this distance. While individual charge values varied depending on location, we typically found partial charge figures of -0.5e for oxygen atoms, +0.3e for hydrogen atoms belonging to hydroxyl groups, $+0.1 \mathrm{e}$ for other hydrogen atoms, and $-0.1 \mathrm{e}$ for carbon atoms. At the layer centered at $2.25 \AA$, a mixture of hydrogen and oxygen atoms was found, and in the next layer (whose atoms are highlighted in Figure 4(a)), mostly hydrogen atoms were found. 
After some layers with mixed composition, we also found a mostly positive layer at $3.75 \AA$ and then another mostly negative layer at $4.75 \AA$. At distances so near the surface, we found that the location of the second nanoparticle had very little influence on the results. For larger radii, we observed a slight long-range modulation in average charge values when the NPs were close - see Figure 4(b). This clustering of like charges in layers prevents the aggregation of nanoparticles within the methanol solution, as Coulombic repulsion between the negative outer layers (at 4.75 Å) should prevent NP agglomeration. Finally, in Figure 4(c), we examined how atomic composition is evolved in time in the layer at $2.75 \AA$. Notice that the composition is steady, outside of slight fluctuations, ensuring the long-term stability of the electrostatic barriers that separate nanoparticles. It is to be noted here that the above formalism works only for nanoparticles when electrostatic force and Van der Waals force overcome the gravitational force (movie is included in supporting information). Therefore, the nanoparticles formation below 10 $\mathrm{nm}$ is also the main challenge for longer stabilization.

\section{Conclusion}

The present investigation of nanoparticles formation by cryo-milling reveals that the free particles have a native surface (virgin surface bounded by dangling bond), which can easily disperse in polar solvents. The dispersion of NPs in solvent makes them uniformly dispersed as free for longer times due to high repulsion force between two nanoparticles. The highest repulsion force between two particles induced due to physisorption of the methanol molecules over the native surface of nanoparticles, which is responsible for the formation of electrical double layer (EDL) and similar charge over the slipping plane. This similar charge is strongly repelled and hence, can reduce the tendency of agglomeration in the polar solvents. The above 
study demonstrates a generic method to synthesis highly concentrated metallic colloids, which can be potentially useful for different applications starting from electronic, energy and biomedical.

\section{Associated Content:}

Supporting Information: Temperature effect in cryomill, TEM Image size distribution (histogram), cold weld ability of metallic nanoparticles and DLVO theory detailed calculation, Molecular dynamics simulation results.

\section{Acknowledgment}

Authors would like to thank SERB-DST for the funding to carry out this research work. LDM acknowledges the Brazilian Research Agencies CNPq and CAPES for financial support.

\section{References}


1. Bakshi, M. S.; Possmayer, F.; Petersen, N. O., Simultaneous Synthesis of Au and Cu Nanoparticles in Pseudo-Core-Shell Type Arrangement Facilitated by DMPG and 12-6-12 Capping Agents. Chem. Mater. 2007, 19 (6), 1257-1266.

2. Bhaumik, J.; Thakur, N. S.; Aili, P. K.; Ghanghoriya, A.; Mittal, A. K.; Banerjee, U. C., Bioinspired Nanotheranostic Agents: Synthesis, Surface Functionalization, and Antioxidant Potential. ACS Biomater. Sci. Eng. 2015, 1 (6), 382-392.

3. Tanner, E. E. L.; Sokolov, S. V.; Young, N. P.; Batchelor-McAuley, C.; Compton, R. G., Fluorescence Electrochemical Microscopy: Capping Agent Effects with Ethidium Bromide/DNA Capped Silver Nanoparticles. Angew. Chem. Int. Ed. 2017, 56 (41), 12751-12754.

4. Pensado, A. S.; Pádua, A. A. H., Solvation and Stabilization of Metallic Nanoparticles in Ionic Liquids. Angew. Chem. Int. Ed. 2011, 50 (37), 8683-8687.

5. Xu, M.; Jiang, J.; Pei, X.; Song, B.; Cui, Z.; Binks, B. P., Novel Oil-in-Water Emulsions Stabilised by Ionic Surfactant and Similarly Charged Nanoparticles at Very Low Concentrations. Angew. Chem. Int. Ed. 2018, 57 (26), $7738-7742$.

6. Min, Y.; Akbulut, M.; Kristiansen, K.; Golan, Y.; Israelachvili, J., The role of interparticle and external forces in nanoparticle assembly. Nat. Mater. 2008, 7, 527.

7. Wu, L.; Zhong, Q.; Yang, D.; Chen, M.; Hu, H.; Pan, Q.; Liu, H.; Cao, M.; Xu, Y.; Sun, B.; Zhang, Q., Improving the Stability and Size Tunability of Cesium Lead Halide Perovskite Nanocrystals Using Trioctylphosphine Oxide as the Capping Ligand. Langmuir 2017, 33 (44), 12689-12696.

8. Ortiz, N.; Skrabalak, S. E., On the Dual Roles of Ligands in the Synthesis of Colloidal Metal Nanostructures. Langmuir 2014, 30 (23), 6649-6659.

9. Saterlie, M. S.; Sahin, H.; Kavlicoglu, B.; Liu, Y.; Graeve, O. A., Surfactant Effects on Dispersion Characteristics of Copper-Based Nanofluids: A Dynamic Light Scattering Study. Chem. Mater. 2012, 24 (17), 32993306.

10. Selvakannan, P. R.; Mandal, S.; Phadtare, S.; Pasricha, R.; Sastry, M., Capping of Gold Nanoparticles by the Amino Acid Lysine Renders Them Water-Dispersible. Langmuir 2003, 19 (8), 3545-3549.

11. Taguchi, T.; Isozaki, K.; Miki, K., Enhanced Catalytic Activity of Self-Assembled-Monolayer-Capped Gold Nanoparticles. Adv. Mater. 2012, 24 (48), 6462-6467. 
12. Willert, M.; Rothe, R.; Landfester, K.; Antonietti, M., Synthesis of Inorganic and Metallic Nanoparticles by Miniemulsification of Molten Salts and Metals. Chem. Mater. 2001, 13 (12), 4681-4685.

13. Kumar, N.; Biswas, K.; Gupta, R. K., Green synthesis of Ag nanoparticles in large quantity by cryomilling. RSC Advances 2016, 6 (112), 111380-111388.

14. Barai, K.; Tiwary, C. S.; Chattopadhyay, P. P.; Chattopadhyay, K., Synthesis of free standing nanocrystalline Cu by ball milling at cryogenic temperature. Mater. Sci. Eng. A. 2012, 558 (0), 52-58.

15. Kumar, N.; Biswas, K., Cryomilling: An environment friendly approach of preparation large quantity ultra refined pure aluminium nanoparticles. J. Mater. Res. Technol. 2017.

16. Zhu, J.; Hersam, M. C., Assembly and Electronic Applications of Colloidal Nanomaterials. Adv. Mater. 2017,29(4), 1603895.

17. Kim, J.; Park, Y.-S.; Veriansyah, B.; Kim, J.-D.; Lee, Y.-W., Continuous Synthesis of Surface-Modified Metal Oxide Nanoparticles Using Supercritical Methanol for Highly Stabilized Nanofluids. Chem. Mater. 2008, 20 (20), 6301-6303.

18. Nandasiri, M. I.; Liu, J.; McGrail, B. P.; Jenks, J.; Schaef, H. T.; Shutthanandan, V.; Nie, Z.; Martin, P. F.; Nune, S. K., Increased Thermal Conductivity in Metal-Organic Heat Carrier Nanofluids. Sci. Rep. 2016, 6, 27805.

19. Jimenez de Aberasturi, D.; Montenegro, J.-M.; Ruiz de Larramendi, I.; Rojo, T.; Klar, T. A.; AlvarezPuebla, R.; Liz-Marzán, L. M.; Parak, W. J., Optical Sensing of Small Ions with Colloidal Nanoparticles. Chem. Mater. 2012, 24 (5), 738-745.

20. Na, H. B.; Song, I. C.; Hyeon, T., Inorganic Nanoparticles for MRIContrast Agents. Adv. Mater. 2009, 21 (21), 2133-2148.

21. Magin, C. M.; Cooper, S. P.; Brennan, A. B., Non-toxic antifouling strategies. Mater. Today 2010, 13 (4), $36-44$.

22. Sen, T.; Bruce, I. J., Surface engineering of nanoparticles in suspension for particle based bio-sensing. Sci. Rep. 2012, 2, 564 .

23. Derjaguin, B., On the repulsive forces between charged colloid particles and on the theory of slow coagulation and stability of lyophobe sols. Trans. Faraday Soc. 1940, 35 (0), 203-215.

24. Kumar, N.; Biswas, K., Fabrication of novel cryomill for synthesis of high purity metallic nanoparticles. Rev. Sci. Instrum. 2015, 86 (8), 083903-8. 
25. Van Duin, A. C. T.; Dasgupta, S.; Lorant, F.; Goddard Iii, W. A., ReaxFF: A reactive force field for hydrocarbons. J. Phys. Chem. A 2001, 105 (41), 9396-9409.

26. Monti, S.; Li, C.; Carravetta, V., Reactive dynamics simulation of monolayer and multilayer adsorption of glycine on Cu(110). J. Phys. Chem. C 2013, 117 (10), 5221-5228.

27. Plimpton, S., Fast Parallel Algorithms for Short-Range Molecular Dynamics. J. Comput. Phys. 1995, 117 (1), 1-19.

28. Aktulga, H. M.; Fogarty, J. C.; Pandit, S. A.; Grama, A. Y., Parallel reactive molecular dynamics: Numerical methods and algorithmic techniques. Parallel Computing 2012, 38 (4-5), 245-259.

29. Linic, S.; Aslam, U.; Boerigter, C.; Morabito, M., Photochemical transformations on plasmonic metal nanoparticles. Nat Mater 2015, 14 (6), 567-576.

30. Rak, M. J.; Friscic, T.; Moores, A., One-step, solvent-free mechanosynthesis of silver nanoparticle-infused lignin composites for use as highly active multidrug resistant antibacterial filters. RSC Advances 2016, 6 (63), 58365-58370.

31. Hunter, R. J.; Ottewill, R. H.; Rowell, R. L., Zeta Potential in Colloid Science: Principles and Applications. Elsevier Science: 1981.

32. Verwey, E. J. W.; Overbeek, J. T. G., Theory of the Stability of Lyophobic Colloids. Dover Publications: 1948.

33. Wang, L.; Albouy, P.-A.; Pileni, M.-P., Synthesis and Self-Assembly Behavior of Charged Au Nanocrystals in Aqueous Solution. Chem. Mater. 2015, 27 (12), 4431-4440. 
Figure
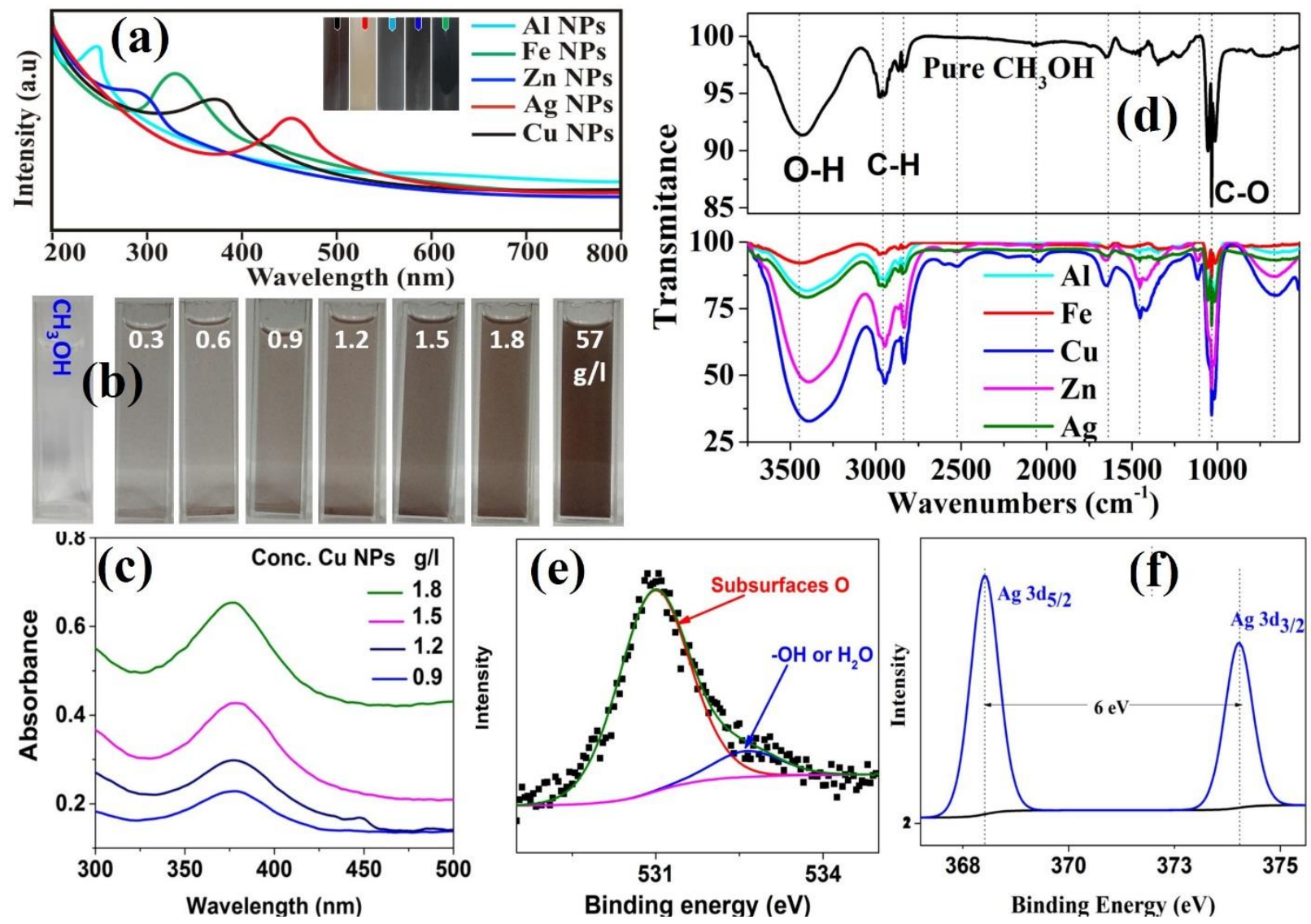

Figure1: (a) UV-spectra of metal nanoparticles dispersed in pure methanol; inset shows different NPs in methanol; (b)optical image of $\mathrm{Cu}$ NPs dispersed in $\mathrm{CH}_{3} \mathrm{OH}$ with different concentration; (c) UV-spectra of $\mathrm{Cu}-\mathrm{NPs}$ dispersed with varying concentration; (d)FTIR spectra of NPs dispersed in $\mathrm{CH}_{3} \mathrm{OH}$; (e)XPS spectra of O Corresponding to Ag NPs; (f) XPS spectrum Ag NPs 

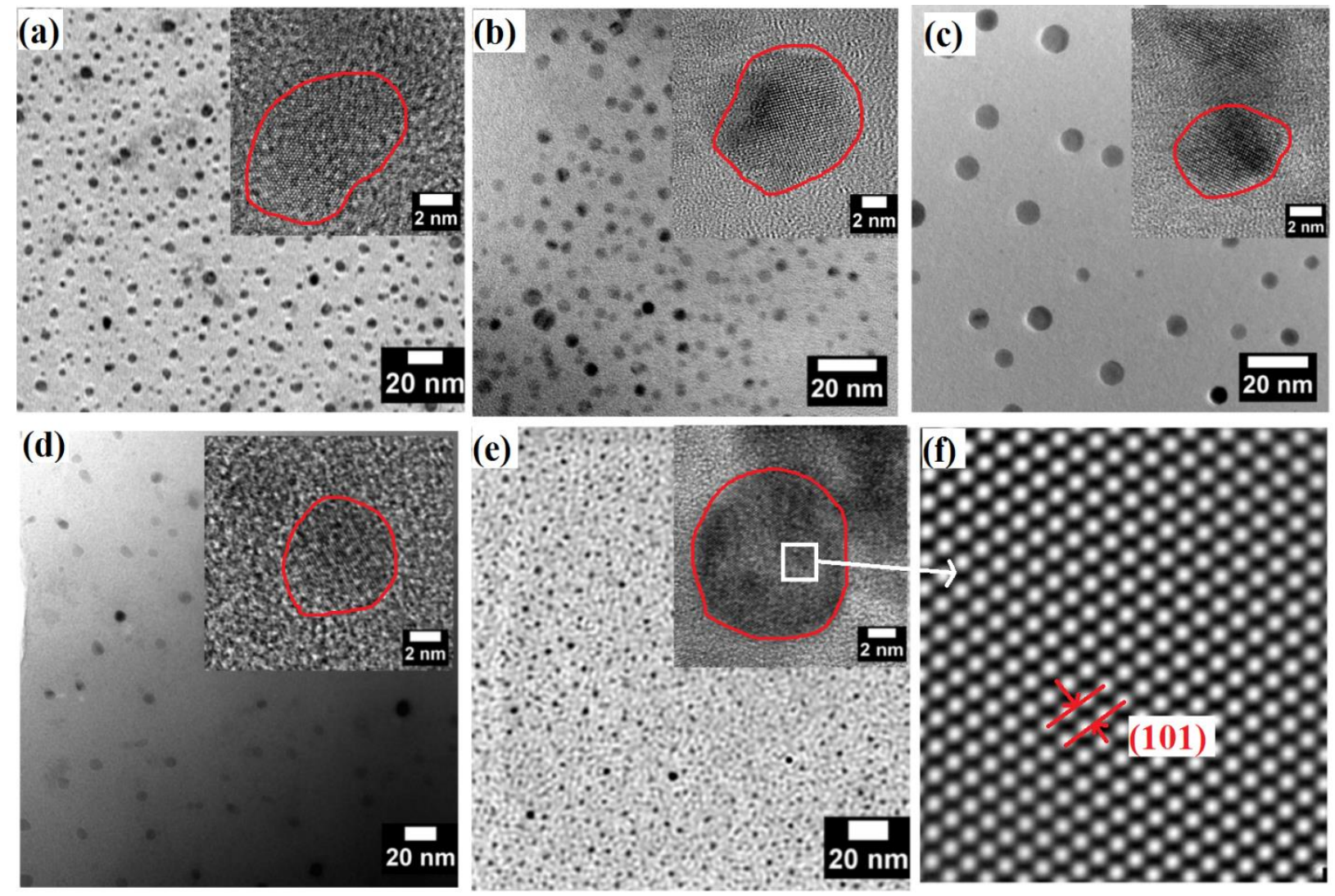

Figure 2: Bright field -Transmission electron microscopic images with inset showing high resolution image(a) Ag NPs; (b) Al-NPs; (c) Cu-NPs; (d) Fe NPs; (e) Zn NPs; (f) FFT filtered image of Zn NP. 

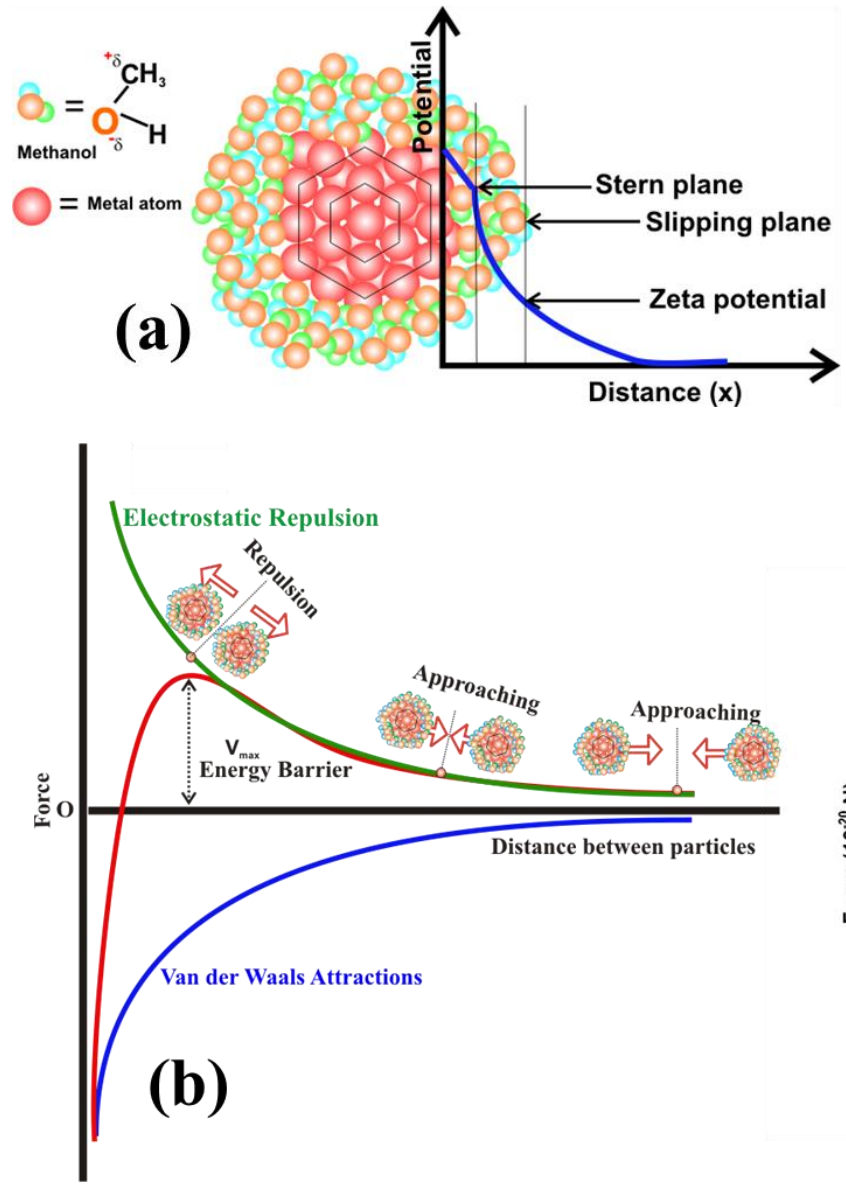
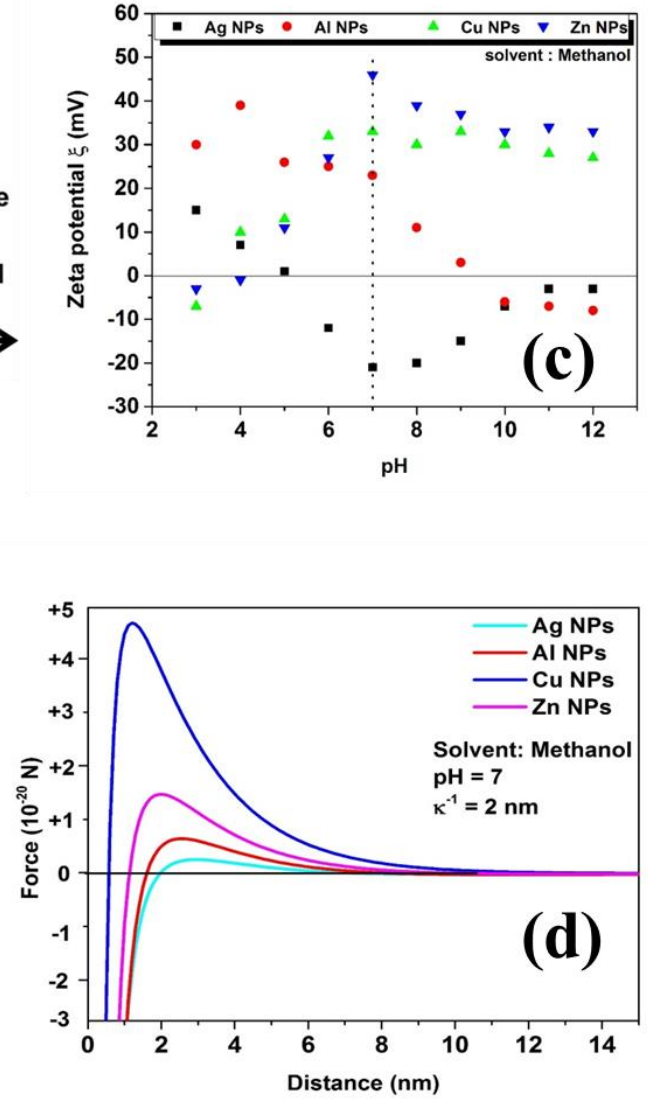

Figure 3: (a) Metal-solvent interaction (EDL formation) and (b) Schematic diagram of NP-NP interaction force profile in the polar solvent; (c) Zeta potentials of the metallic NPs dispersed in methanol with varying $\mathrm{pH}$ (d) Calculated force profiles of $\mathrm{Ag}, \mathrm{Al}, \mathrm{Cu}$ and $\mathrm{Zn}$ NPs at $7 \mathrm{pH}$ and 2 nm Debye length $\left(\kappa^{-1}\right)$. 


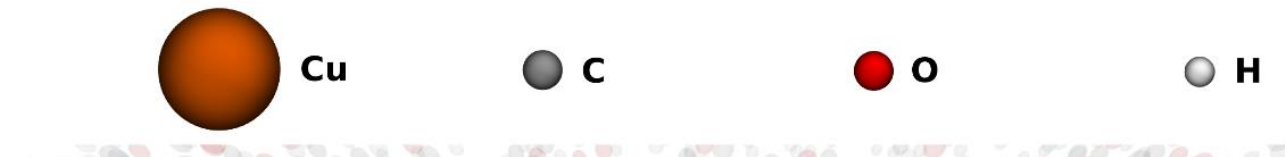

(a)
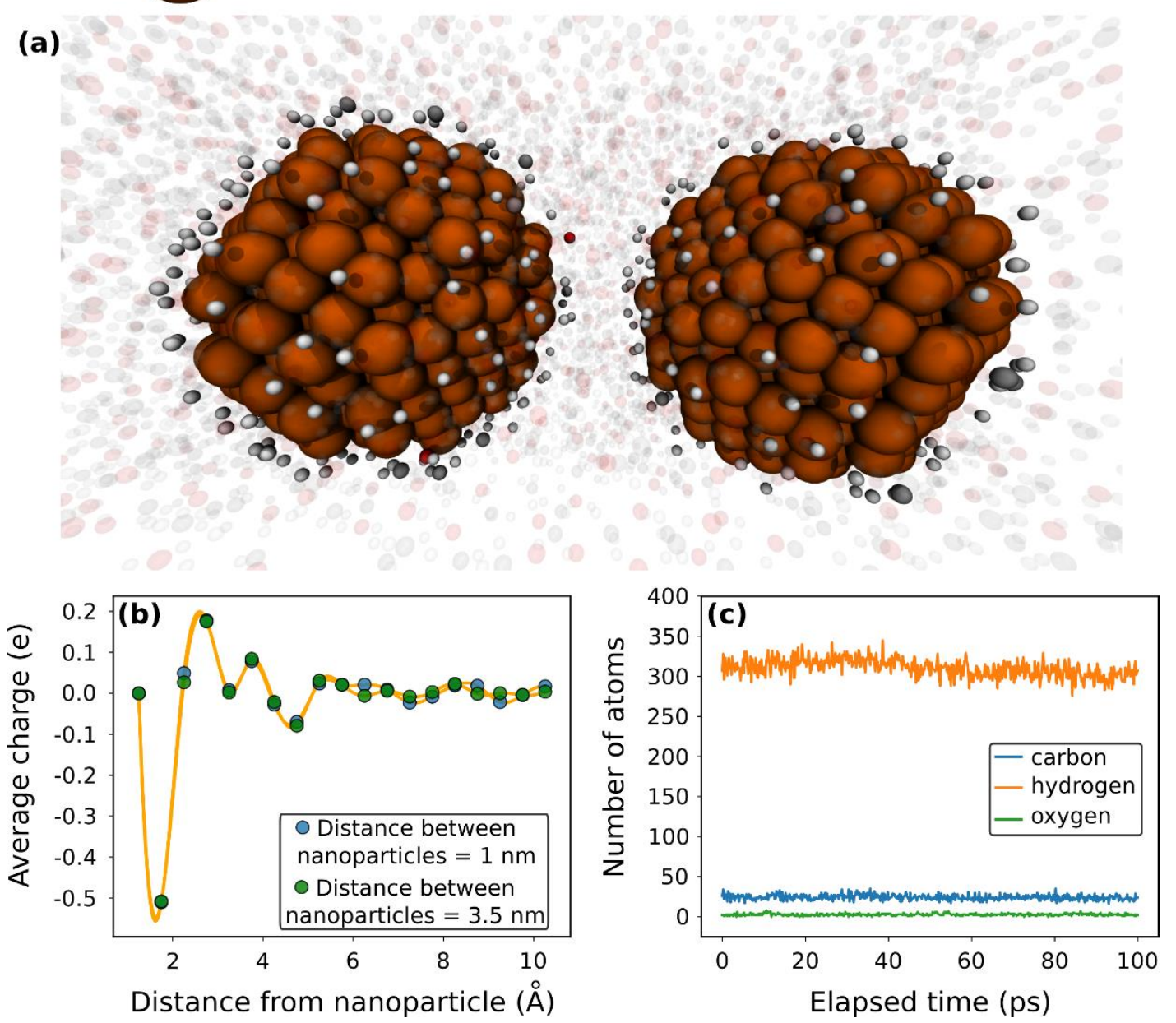

Figure 4: (a) Snapshot at the end of an MD equilibration process, for a system where the copper nanoparticles are $1 \mathrm{~nm}$ apart. Most solvent atoms are transparent to aid visualization, with the exception of those located within 2.5 - 3.0 $\AA$ of the nanoparticle surface. (b) Graph displaying average charge values at a distance from the nanoparticle surface, for two NP separation values. For instance, to obtain the data point at $2.75 \AA$, we considered in the average all opaque atoms from (a). The yellow curve is merely a guide to the eye. (c)Graph is presenting the number of atoms located within $2.5-3.0 \AA$ of the nanoparticle surface versus elapsed simulation time, for copper NPs $1 \mathrm{~nm}$ apart. Notice the stability in atomic composition. 
Table 1: Size of metals nanoparticles and position of surface plasmon resonance (SPR) dispersed in methanol.

\begin{tabular}{ccc}
\hline Metal NPs & Size $(\mathbf{n m})$ & $\begin{array}{c}\text { SPR band }\left(\boldsymbol{\lambda}_{\text {max }}\right) \\
\text { (Methanol) }\end{array}$ \\
\hline $\mathrm{Ag} \mathrm{NPs}$ & $6 \pm 2$ & 465 \\
\hline $\mathrm{Al} \mathrm{NPs}$ & $6 \pm 3$ & 266 \\
\hline $\mathrm{Cu}$ NPs & $7 \pm 2$ & 375 \\
\hline Zn NPs & $3 \pm 1$ & 274 \\
\hline Fe NPs & $8 \pm 3$ & 310 \\
\hline
\end{tabular}




\section{TOC}

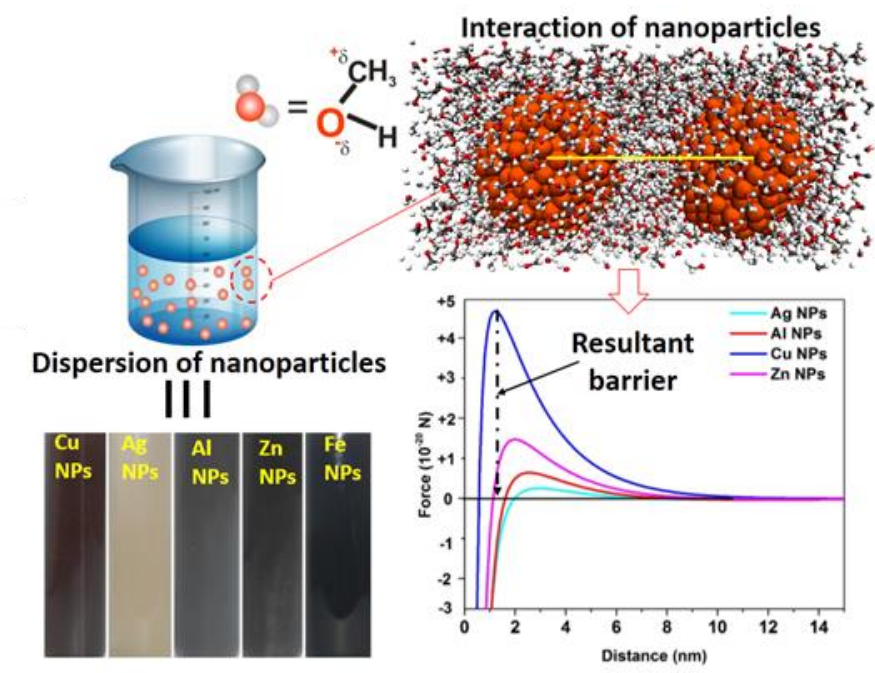

